\title{
Pernicious Anaemia with Normal Vitamin B12
}

\author{
João Tavares, Bernardo Baptista, Bebiana Gonçalves, Alexandra Bayão Horta \\ Medicine Department, Hospital da Luz, Lisbon, Portugal
}

Received: 04/01/2019

Accepted: 09/01/2019

Published: $18 / 02 / 2019$

How to cite this article: Tavares J, Baptista B, Gonçalves B, Horta AB. Pernicious anaemia with normal vitamin B12. EJCRIM 2019;6: doi:10.12890/2019_001045.

Conflicts of Interests: The Authors declare that there are no competing interests.

This article is licensed under a Commons Attribution Non-Commercial 4.0 License

\section{ABSTRACT}

A 49-year-old female patient presented to our hospital with asthenia, odynophagia, low grade fever, worsening symptoms of chronic depression, and symmetric leg paresthesias. Investigations showed macrocytic anaemia, leucopenia, thrombocytopenia, high lactate dehydrogenase levels and a normal Coombs test. Trilineage dysplasia was detected in the bone marrow biopsy specimen. The diagnostic work-up led us to the diagnosis of pernicious anaemia with a spuriously normal value of vitamin B12 and high titres of anti-intrinsic factor autoantibodies. This case highlights the importance of considering vitamin B12 deficiency in the differential diagnosis of myelodysplasia, even when vitamin B12 levels seem to be normal.

\section{LEARNING POINTS}

- Vitamin B12 deficiency is characterized by neuropsychiatric manifestations and bone marrow failure with accompanying dysplastic changes.

- Spuriously normal vitamin B12 levels can occur in pernicious anaemia due to anti-intrinsic factor autoantibody interference in the laboratory assay.

- Myelodysplastic syndromes and vitamin B12 deficiency share clinical and laboratory similarities, so a correct differential diagnosis is crucial for adequate treatment.

\section{KEYWORDS}

Anti-intrinsic factor antibodies, myelodysplastic syndrome, pernicious anaemia, vitamin B12 deficiency, spuriously normal vitamin B12

\section{CASE DESCRIPTION}

We present the case of a 49-year-old female patient with a history of psoriasis vulgaris and chronic depression, who was admitted to our hospital with a 7-day history of asthenia, odynophagia and low-grade fever. She recalled worsening of depressive symptoms and symmetric leg paresthesias during the previous year. Current medication included brotizolam and a topical corticosteroid. There was no history of alcohol consumption. A complete physical examination was unremarkable except for skin and conjunctiva pallor, and a regular tachycardia (116 beats per minute). Laboratory tests revealed pancytopenia with hypoproliferative macrocytic anaemia: haemoglobin $7.3 \mathrm{~g} / \mathrm{dl}$ (normal range $12-16 \mathrm{~g} / \mathrm{dl}$ ), mean cell volume $111 \mathrm{fl}$ (normal range 80-100 fl), mean corpuscular haemoglobin $39 \mathrm{pg} / \mathrm{cell}$ (normal range 26-34 pg/ cell), red cell distribution width $23 \%$ (normal range $11.5-14.5 \%$ ), reticulocyte production index 0.37 , white blood cells $1,930 \times 10^{\%} / /$ with an absolute neutrophil count of $560 \times 10^{\%} / \mathrm{l}$, and platelets $135,000 \times 10^{\%} / \mathrm{I}$. The blood smear revealed anisocytosis and poikilocytosis. The erythrocyte sedimentation rate was $67 \mathrm{~mm} / \mathrm{h}$ (normal range $<25 \mathrm{~mm} / \mathrm{h}$ ) and the serum lactate dehydrogenase (LDH) level was markedly elevated at 2,668 IU/I (normal range 81-234 IU/I). The direct Coombs test and bilirubin levels were normal. Serum creatinine, liver function tests and thyroid function tests were all within normal limits, as were the coagulation times and the serum ferritin, folate and vitamin B12 
levels (387, normal range 142-724 pmol/I). Diagnostic work-up of a bone marrow aspirate and biopsy showed moderate signs of trilinear dysplasia. The anti-intrinsic factor autoantibody was positive $(92.9 \mathrm{U} / \mathrm{ml})$ with a titre two to three-fold the upper limit of normal, while the antiparietal cell antibody was negative. Upper gastrointestinal endoscopy showed gastric fundus and body atrophy with an unspecific chronic gastritis on histological examination. Electromyography of the lower limbs was normal. During the patient's admission, the odynophagia and low-grade fever remitted spontaneously. Intra-muscular cyanocobalamin was started at $1 \mathrm{~g}$ once daily for 1 week, followed by $1 \mathrm{~g}$ weekly for a month and $1 \mathrm{~g}$ every other month thereafter, with an excellent bone marrow response (Fig. 1) characterized by marked reticulocytosis, prompt haemoglobin recovery and a rapid decrease in LDH serum levels. There was a noteworthy symptomatic improvement, particularly of the asthenia and neuropsychiatric symptoms. The lower limb paresthesias improved more slowly but finally subsided. The patient remains asymptomatic after 2 years of follow-up.

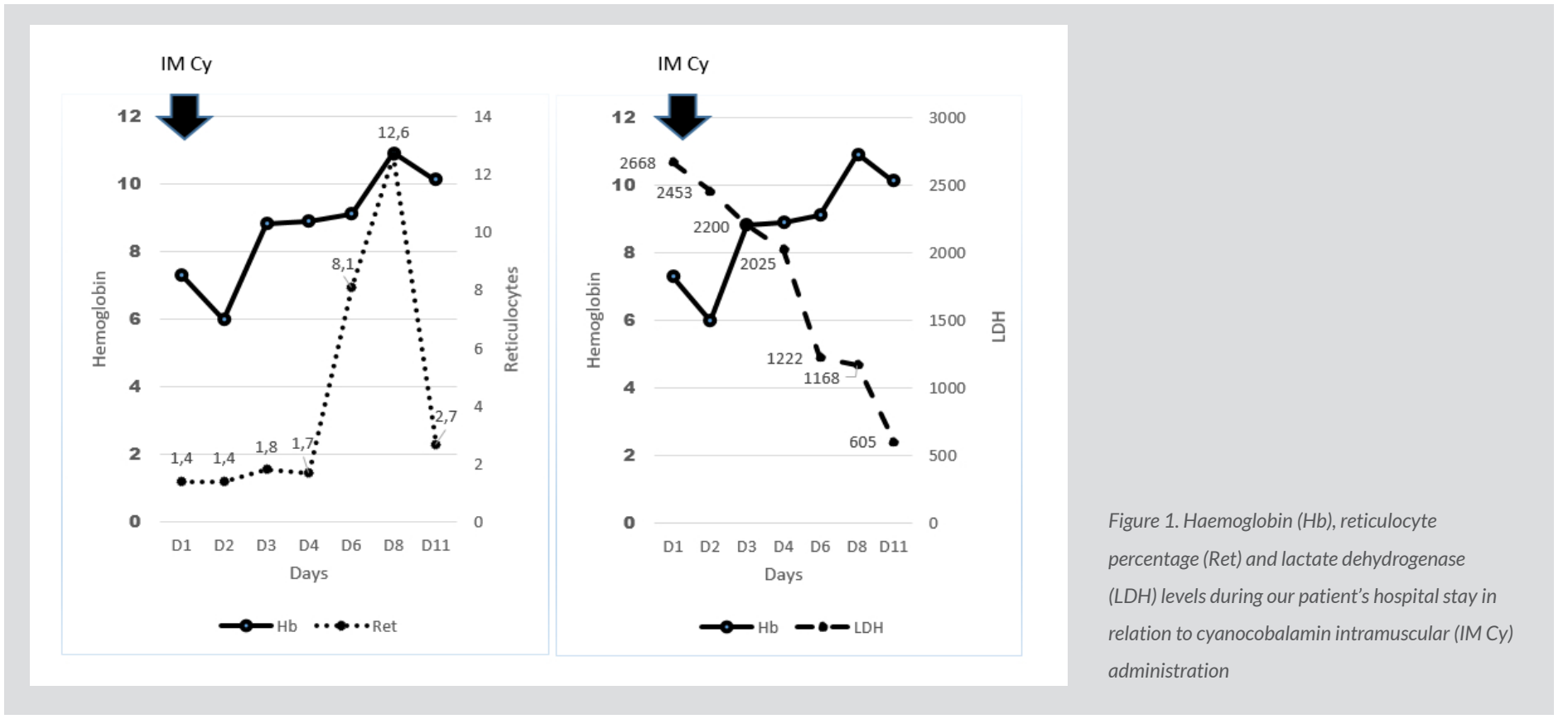

\section{DISCUSSION}

In its classic clinical presentation, vitamin B12 deficiency is accompanied by neuropsychiatric changes and bone marrow failure characterized by dyserythropoiesis with cytopenias and blood cell dysplasia ${ }^{[1]}$. Pernicious anaemia is the most common cause of vitamin B12 deficiency worldwide and is caused by autoimmune gastric parietal cell destruction ${ }^{[2]}$. These cells produce intrinsic factor which binds to ingested vitamin B12, thus enabling its absorption. However, autoimmune parietal cell destruction causes a deficiency of this factor with consequent vitamin B12 malabsorption ${ }^{[2]}$. Pernicious anaemia diagnosis is based on the documentation of low vitamin B12 levels and the presence of anti-intrinsic factor autoantibodies (or anti-parietal cell antibodies) ${ }^{[3]}$. We report a case of pernicious anaemia with a spuriously normal value of vitamin B12. Current automated vitamin B12 assays use an intrinsic factor binding method ${ }^{[3]}$. Therefore, the presence of high titres of antibodies to intrinsic factor, which was the case in our patient, can interfere with the assay, resulting in spuriously normal vitamin B12 levels ${ }^{[4]}$. Methylmalonic acid (MMA) and homocysteine (Hcy) are substrates of vitamin B12-dependent enzyme reactions and, in the event of vitamin B12 deficiency, they accumulate and their blood levels increase ${ }^{[3]}$. We decided not to determine MMA and Hcy levels because the clinical presentation and the combination of positive anti-intrinsic factor antibodies with upper endoscopy findings were highly suggestive of pernicious anaemia. Also, these assays were not readily available in our unit. Another way to avoid automated vitamin B12 assay interference is to measure the transcobalamin-bound fraction of plasma B12 (holoTC, also called 'active' B12) ${ }^{[3,5]}$, but this assay is still not used in clinical practice. Finally, a clinical and laboratory response to parenteral cyanocobalamin indicates vitamin B12 deficiency ${ }^{[5]}$, as happened in our case.

Acknowledging the limitations of laboratory measurement of vitamin B12 is crucial, especially as vitamin B12 deficiency is a treatable cause of myelodysplasia. Misdiagnosing a myelodysplastic syndrome based on a normal serum level of vitamin B12 can ultimately lead to unnecessary and harmful consequences for patients. 


\section{REFERENCES}

1. Hunt A, Harrington D, Robinson S. Vitamin B12 deficiency. BMJ 2014;349:g5226

2. Stabler SP Clinical practice. Vitamin B12 deficiency. N Engl J Med 2013;368:149-160.

3. Green R. Vitamin B12 deficiency from the perspective of a practicing hematologist. Blood 2017;129:2603-2611.

4. Yang DT, Cook RJ. Spurious elevations of vitamin B12 with pernicious anemia. N Engl J Med 2012;366:1742-1743.

5. Solomon LR. More on failures of cobalamin assays in pernicious anemia. N Engl J Med 2012;367:1570; author reply 1571. 